\title{
Effect of fiber treatment on dimensional stability and chemical resistance properties of hybrid composites.
}

\begin{abstract}
In this work, oil palm empty fruit bunch (EFB) and jute fibers were treated by 2-hydroxy ethyl acrylate (2-HEA) to enhance interaction with the epoxy matrix in hybrid composites. Hybrid composites were fabricated by the hand lay-up technique by reinforcing chemicaltreated oil palm EFB and jute fibers in an epoxy matrix. Physical (density, void content, water absorption, and thickness swelling) and chemical resistance properties of treated hybrid composites were characterized. Chemically treated oil palm EFB/jute fiber reinforced hybrid composites display better dimensional stability (water absorption and thickness swelling) and chemical resistance as compared to untreated hybrid composites.
\end{abstract}

Keyword: 2-Hydroxy ethyl acrylate (2-HEA); Chemical resistance; Dimensional stability; Hybrid composites; Jute fibers; Oil palm fibers. 